\title{
La interacción deliberativa en los procesos de participación vinculados a las decisiones públicas
}

\author{
Francisco José Francés García \\ Universidad de Alicante. Departamento de Sociología II \\ francisco.frances@ua.es
}

Recepción: 10-09-2014

Aceptación: 16-06-2016

\section{Resumen}

El artículo propone un análisis de la significación participativa y la comunicación deliberativa presentes en los procesos de participación ciudadana vinculados a las decisiones públicas. El principal objetivo radica en analizar en qué condiciones se produce la deliberación y qué efectos cognitivos y valorativos produce en los participantes, con el fin de articular decisiones colectivas y acción institucional. Como soporte pragmático y empírico de las ideas trabajadas, se utiliza la figura del presupuesto participativo, que constituye un espacio de participación orientado a la propuesta, deliberación y toma de decisiones relacionadas con la política pública. El texto concluye señalando la importancia de aspectos como la valoración de la solidaridad y la democracia interna en las asambleas ciudadanas, la valoración del tiempo y la información disponibles para el debate o la percepción del grado de compromiso institucional como factores que influyen en la calidad deliberativa de procesos participativos ligados a las decisiones públicas.

Palabras clave: participación; democracia; deliberación; acción pública; presupuesto participativo

\section{Abstract. Deliberative Interaction in Participation Processes Concerning Public Decisions}

This article analyzes the participatory significance and deliberative communication that must be present in citizen participation processes concerning public decisions. The main purpose is to examine the conditions in which deliberation is produced and the cognitive and appreciatory effects of deliberation on the participants in order to articulate collective decisions and institutional action. Participatory budgeting is used as a pragmatic and empirical framework for the ideas under consideration. This experience constitutes a sphere for participation oriented toward proposals, deliberation and decision making on public policy. The paper concludes that aspects such as the assessment of solidarity and internal democracy within citizen assemblies, time and information for the debate or the degree of institutional commitment are important factors that influence the deliberative quality of participative processes concerning public decisions.

Keywords: participation; democracy; deliberation; public action; participatory budgeting 


\section{Sumario}

\begin{tabular}{|c|c|}
\hline \multirow{3}{*}{$\begin{array}{r}\text { 1. Introducción } \\
\text { 2. Estado de la cuestión. La significación } \\
\text { de la acción participativa en los procesos } \\
\text { deliberativos }\end{array}$} & 4. Análisis y discusión de resultados. \\
\hline & $\begin{array}{l}\text { La valoración de las condiciones } \\
\text { de la deliberación }\end{array}$ \\
\hline & 5. Conclusiones \\
\hline 3. Datos y metodología & Referencias bibliográficas \\
\hline
\end{tabular}

\section{Introducción}

Dentro del planteamiento teórico de los procesos participativos, el papel que desempeñan los ciudadanos plantea siempre un terreno resbaladizo, por cuanto inciden multitud de factores que van desde los campos de estudio nítidamente psicológicos, cercanos a las motivaciones y a las predisposiciones individuales, hasta las esferas de análisis institucional encargadas de sistematizar los procesos de participación plenamente cristalizados. La conjunción de factores micro y macro, el lógico solapamiento de diversas disciplinas científicas o la complejidad que encierra en este campo el uso de conceptos que, por su naturaleza, son difícilmente categorizables hace que el reto de comprender la potencialidad de los ciudadanos individuales dentro de la acción pública se convierta en un ejercicio complicado, que se acentúa por cuanto cualquier premisa de análisis debe partir del reconocimiento de la complejidad social existente. Un análisis que, además, se torna necesario, por cuanto constituye un campo de investigación que trasciende al mero debate académico para situarlo dentro del pragmatismo participativo vinculado a las políticas públicas. Esta traslación se concreta en lo que algunos autores han dado en llamar un nuevo modelo de participatory institutional arrangements (DeCaro y Stokes, 2013: 8; Dover y Lawrence, 2010: 306) o participatory governance arrangements (Bassoli, 2012: 1186; Wampler, 2012: 341), dentro del sentido amplio del concepto de empowered participatory governance desarrollado por Fung y Wright (2001, 2003; Fung, 2004) como diseño institucional ideal propuesto para la toma deliberativa de decisiones y la solución pragmática de problemas específicos entre participantes sobre un tema de interés común (Baiocchi y Ganuza, 2014: 32).

Una de las características de las sociedades complejas modernas es la existencia de una sociedad civil heterogénea que se presenta en la dimensión pública de la acción social a través de una multitud de formas de participación, en las que confluyen la agregación y la integración de preferencias particulares. Esta pluralidad de formas e intereses constituye la base conceptual de la que parte la concepción deliberativa de la democracia, así como su "giro empírico» (Bächtiger et al., 2007: 485; Hansen y Rostboll, 2012: 508; Beste, 2013: 2), en busca de las condiciones que favorezcan el despliegue pragmático de las premisas propias de la democracia deliberativa. El enfoque resultante de estas premisas contempla las contingencias que se presentan en los procesos de inte- 
racción participativa, así como los condicionantes que emergen en los espacios que persiguen la integración práctica entre la democracia liberal representativa y la democracia participativa de corte comunitario. Ello abre, sin duda, nuevos horizontes para la discusión y apunta hacia la pertinencia de explorar en profundidad la óptica deliberativa de los procesos de participación en democracia como una estrategia progresiva de reforma institucional. Pero también obliga a estar atentos a las posibles disfunciones, debilidades y problemáticas de orden tanto teórico como práctico, en ocasiones con un considerable calado y unas consecuencias que puede generar este giro institucional (entre otros, Cohen y Fung, 2004: 27; Levine et al., 2005: 3; Dzur, 2008: 35; Engelken-Jorge, 2012: 19).

Entre las nuevas prácticas democráticas de deliberación colectiva vinculadas a la acción pública emerge, en un lugar destacado en las últimas décadas, la figura del presupuesto participativo, que actuará en las próximas páginas como soporte pragmático de las ideas apuntadas en este artículo. El presupuesto participativo es una de las herramientas de democracia directa que pone sobre el tapete de la planificación comunitaria la existencia de fórmulas de cogestión y codecisión entre instituciones y ciudadanía en materias importantes de gobierno municipal. La figura del presupuesto participativo se ha presentado, durante los últimos años, en muchas regiones y ciudades europeas como una iniciativa que pretende implicar activamente a la ciudadanía en las decisiones públicas y desarrollar estrategias efectivas de concertación y legitimación en la acción institucional. Esta experiencia, de fuerte radicación en Latinoamérica, nace en Brasil a finales de la década de 1980 y, a partir de ese momento, presenta un crecimiento significativo no solo en Latinoamérica, sino también en todo el mundo, con lo que, para el año 2010, se pueden identificar más de 1500 experiencias en el planeta (Ganuza y Baiocchi, 2012: 1), más de 200 de las cuales se sitúan en Europa, unas 120 en Asia y casi mil están presentes en Latinoamérica (Sintomer et al., 2013: 3). Para el caso español, aunque de forma variable en su autodefinición y en el alcance real de la deliberación interna (Ganuza y Francés, 2012: 111), se pueden contar aproximadamente casi un centenar de experiencias desde su aparición en 2001 (Pineda y Pires, 2008: 72; Ganuza y Francés, 2012: 67).

A pesar de que la literatura investigadora en torno al presupuesto participativo es ya muy extensa, aún hoy son escasas las investigaciones que se han ocupado en términos pragmáticos del impacto deliberativo interno dentro de estos procesos. En este sentido, cabría destacar algunos trabajos empíricos como los de Avritzer (2000), Talpin (2011), Cardoso et al. (2011) o Ganuza y Francés (2012), que aportan una información muy valiosa en torno a los sesgos y a las asimetrías participativas que acontecen en los presupuestos participativos, sobre todo en términos de caracterización de perfiles sociodemográficos y sociopolíticos, así como el diferencial de oportunidades de influencia de los participantes en el debate colectivo. Existe cierto vacío, sin embargo, en relación con el impacto sobre la deliberación de las condiciones en que se celebran las dinámicas colectivas que acontecen en el proceso. 
Uno de los elementos que incorpora el presupuesto participativo en la mayor parte de sus modelos organizativos es la celebración de reuniones ciudadanas, que se constituyen como espacios comunicativos donde se despliega la interacción deliberativa por parte de los participantes para alcanzar acuerdos en relación con las prioridades de gastos públicos que deben dirimir. Estos espacios generan una dinámica procesual sobre una ciudadanía que, como hemos comentado, cada vez es más heterogénea, con necesidades diferentes $y$, en ocasiones, contrapuestas. $Y$ en el marco operativo que propone el presupuesto participativo, los intereses singulares de la ciudadanía deben integrarse a través de acciones comunicativas, para traducirse finalmente en decisiones colectivas que identifiquen un bien común compartido. De ahí la importancia, la preeminencia y la necesaria consideración que poseen los elementos propios del enfoque deliberativo dentro de este tipo de experiencias de innovación democrática.

El objetivo de este texto descansa, por lo tanto, en la voluntad de analizar, ayudándonos de la experiencia propuesta, en qué condiciones se produce la deliberación y qué efectos cognitivos y valorativos produce en los participantes, siendo el marco para el caso que nos ocupa decisiones participadas en torno a políticas públicas. Para ello, se analizará el alcance pragmático de las diferentes claves explicativas que acontecen en la interacción deliberativa dentro de la figura del presupuesto participativo.

\section{Estado de la cuestión. La significación de la acción participativa en los procesos deliberativos}

La emergencia del enfoque (o de los enfoques sería más correcto) de democracia deliberativa es quizás una de las innovaciones más sugerentes en los tiempos recientes de la teoría democrática. El elemento definitorio de la democracia deliberativa y su fundamento procesual bien podrían residir, como comenta Dryzek (2000: 31), en el hecho de que «los individuos participantes en procesos democráticos son susceptibles de cambiar sus opiniones y preferencias como resultado de la reflexión inducida por la deliberación». No es lugar aquí de enumerar las largas listas de voces favorables o críticas en relación con este enfoque de la democracia, algo que, por otra parte, ya se ha encargado de sintetizar la amplia revisión investigadora existente (ver, entre otros, Bohman, 1996, 1998; Sanders, 1997; Dryzek, 2000; Chambers, 2003; Gutmann y Thompson, 2004; O’Flynn, 2006). Este texto sí hará, en cambio, uso de aquellas aportaciones teóricas que contribuyan a centrar el debate en torno a aquellas cuestiones que definen el marco de la interacción deliberativa.

La democracia deliberativa, sin duda, descansa sobre una idea de la política (Young, 2000: 173) que tiene la aspiración de apuntar hacia la justicia a través de la discusión pública y de la toma de decisiones, que debería teorizar y practicar una alternativa distinta a la que, por un lado, incide en la competencia de intereses privados propia del acercamiento liberal y a la que, por otro lado, deposita el énfasis en la negación de las diferencias en la discusión 
pública del bien común, mucho más ligada a un acercamiento republicano y comunitarista. La alternativa vendría dada por la generación de un proceso de debate público y de una toma de decisiones que busca incluir y reconocer todas las perspectivas de los diferentes segmentos o grupos sociales llamados a la deliberación, haciendo para ello uso del conocimiento, la interacción y la información como fuentes de comprensión para alcanzar consensos satisfactorios. Desde aquí, la democracia deliberativa trazaría nexos hacia la idea que desarrolla Benjamin Barber (1998) cuando habla de la deseabilidad de la construcción de democracias fuertes, que pasarían por la revitalización de una forma de comunidad que no es colectivista (reconocimiento de las diferencias), por una forma de razonamiento público que no es conformista (deliberación crítica) y por un conjunto de instituciones cívicas compatibles con las sociedades complejas (adaptación y compromiso institucional).

Vista así, la deliberación se aleja mucho de la idea de la mera agregación de intereses y, paralelamente, desborda los enfoques más reduccionistas de la democracia participativa, en los que prima únicamente la afluencia de los sujetos en la arena pública para la toma de decisiones colectivas, aunque no por ello rehúye en ningún momento la necesaria concurrencia de todos los actores afectados por una decisión pública. La perspectiva deliberativa de la democracia parte de lo que se ha dado en llamar "política de la presencia» (Phillips, 1995), es decir, de los procesos que centran sus esfuerzos en los propios mensajeros, descansando la representación en las identidades de los sujetos que concurren en el proceso deliberativo. En otras palabras, mientras que la democracia representativa invoca la noción de una re-presentación funcional o ideológica, la democracia deliberativa alude a la idea de la presentación social como fuente de generación de poder legítimo. Desde esta perspectiva, siguiendo a Barber, los términos participación, deliberación y comunidad son aspectos de un único modo de ser social: la ciudadanía. La comunidad sin participación ni deliberación genera, en primer lugar, consenso irreflexivo y uniformidad, y, en segundo lugar, alimenta una conformidad coercitiva presidida por lo que podríamos llamar «un colectivismo unitario». Por otra parte, la participación sin comunidad nos aboca a una lucha de intereses competitiva y desorientada que reafirma la negación de la propia idea de comunidad.

La óptica deliberativa de la política se funda también en el abandono de la dicotomía ciudadana clásica establecida por Stuart Mills, que dividía a los ciudadanos de una forma mecánica entre choosers, es decir, ciudadanos formados, informados y con unas preferencias claras en torno a sus intereses dentro de los procesos democráticos, y learners, ciudadanos que se hallan carentes de información y, por lo tanto, con falta de capacitación para formarse opiniones fundadas en torno a los intereses públicos. Queda apartada aquí cierta concepción elitista schumpeteriana o ilustrada de la participación que establece la necesaria primacía en el debate de unos actores sobre otros, ya sean estos individuos colectivos ciudadanos o instituciones. Lejos de estos parámetros especulativos, pensados sin duda para ámbitos deliberativos que desbordan el ámbito de proximidad cotidiano y que introducen claves prácticas de distinta naturaleza 
(Nanz y Steffek, 2004), pero reconociendo las dificultades que el ejercicio de la ciudadanía entraña, podríamos hablar de que la democracia deliberativa es la política de los «aficionados», y ello en una doble vertiente: aficionados por cuanto cada persona es llamada a encontrarse con otras sin la intermediación de expertos, lo cual no quiere decir en absoluto que estén carentes de información y cualificación, como luego comprobaremos. Aficionados, también desde el componente más etimológico del término, por cuanto se presume la "afición» de los participantes por la estimación o, al menos, la consideración de lo que debe ser el bien común a todos los miembros de una comunidad política. Los sujetos concurren, así, en situación de simetría táctica en el espacio generado para el debate público, que, en el caso de los procesos tratados en este artículo, constituyen lugares participativos orientados a la propuesta, a la deliberación y a la decisión relacionados con las decisiones de gasto público, incorporando iniciativas e intereses que son dirimidos a través del propio proceso.

Procesos de innovación democrática como el del presupuesto participativo poseen, por lo tanto, la capacidad, o al menos la potencialidad, de articular la incompletud inicial de las preferencias individuales (dimensión agregativa, más cercana a la visión liberal) con el reconocimiento y la conjugación de los diferentes espectros de estas en un espacio común (dimensión integrativa, más cercana a la visión republicana), donde los individuos no pertenecerían ya tanto a grupos singulares como a redes sociales, las cuales toman forma (que no existencia) en el devenir intercomunicativo del proceso. Es en el marco de interacción de esas redes donde cada persona fragua sus necesidades, prioriza intereses y aborda conflictos.

Bajo esta óptica, cada persona define la realidad de manera particular, pero, en lugar de adherirse a significantes propuestos por agentes externos al proceso participativo, cada sujeto tiene la oportunidad de conocer cómo el resto de sujetos definen su realidad, y tanto la legitimidad de los procesos como la valoración de sus resultados se desprenden de razones cuya validez ya solo podría juzgarse si se dejara de lado la posición de observador externo o experto y se asumiera la participación en el intercambio de argumentaciones del resto de participantes (Colombo, 1998; Barreiro, 2012: 3). En definitiva, la legitimidad del proceso, como argumenta Habermas (1998) en su teoría de la acción comunicativa, depende, en último término, de un acuerdo comunicativo. Y la realidad que resulta de este acuerdo comunicativo es construida socialmente. Por todo ello, se puede decir que la deliberación (y la interacción comunicativa como su materia prima) instituye la realidad social, le da forma, le otorga sentidos compartidos, lo que, en un sentido más amplio, incorpora a la intersubjetividad como eje vertebrador en la construcción comunicativa de la realidad social (Berger y Luckmann, 1993: 191). Hablamos, por lo tanto, de un enfoque de la democracia en el que poseen un peso fundamental la deliberación, la comunicación y la participación como elementos definidores de la política (cristalizada esta como construcción social de la realidad), que opera desde un nivel mucho más exigente que el que se presenta habitualmente. 
Cuando, en un presupuesto participativo, un ciudadano participa en una dinámica de trabajo o presenta una propuesta en una asamblea, esta, obviamente, posee un carácter denotativo, orientado a la satisfacción de la necesidad enunciada, pero también, y de forma inseparable, la propia propuesta se convierte en una acción política a través de la cual el propio ciudadano asoma un horizonte de significados que, ineludiblemente, son interpretados por el resto de participantes, lo cual genera en unos u otros comprensión, apoyo, indiferencia, rechazo o conflicto. En este sentido, como sostiene Barber, la deliberación no se circunscribe únicamente al ámbito de la intersubjetividad comunicativa, aunque lo incorpora como instancia práctica. De tal suerte, la acción participativa en este tipo de procesos presenta una significación que entronca con el entramado de subjetividades compartidas y elaboradas culturalmente.

Y esta es precisamente una de las razones de ser de procesos participativos de innovación democrática como los presupuestos participativos en relación con su finalidad pragmática. Si la definición y la priorización de necesidades fuera en verdad natural, objetiva y prepolítica, no sería necesario desplegar un proceso en el que se persigue la concurrencia de sujetos en escenarios colectivos (ya sean estos asambleas, dinámicas, reuniones o encuentros) para debatir, deliberar y decidir, en definitiva, para generar heterorreflexividad. Bastaría con aplicar un esquema de racionalidad preestablecida en la jerarquización de las necesidades planteadas, desestimando la aportación de un proceso participativo que vendría, en este caso, a confundir o a distorsionar las decisiones objetivamente aconsejables. Lo cierto es que, más allá de las necesidades elementales de subsistencia, estas cristalizan a través de su definición social, lo cual nos ayuda mucho a comprender el continuo debate y la incomodidad escénica que se presenta dentro de los procesos participativos entre el interés singular y la responsabilidad social, cuestión que toma especial relevancia si los procesos están orientados hacia la decisión por parte de los propios ciudadanos de cuestiones relacionadas con la acción pública.

\section{Datos y metodología}

A fin de profundizar en el objetivo propuesto en el artículo, esto es, analizar en qué condiciones se produce la deliberación y qué efectos cognitivos y valorativos produce en los participantes del presupuesto participativo, se ha optado por acompañar la discusión teórica con un análisis empírico de carácter cuantitativo y estadístico. Para ello, se ha hecho uso de los datos procedentes de una encuesta realizada en seis países (España, Francia, Reino Unido, Alemania, Italia y Brasil) a participantes de 17 experiencias de presupuestos participativos. Dicha encuesta fue coordinada en 2011 por el IESA-CSIC dentro del proyecto Democracia, participación y espacio político: Un estudio comparado, financiado por el Ministerio de Educación y Ciencia dentro del Plan Nacional de I+D+I. Quede aquí mi agradecimiento al IESA por las facilidades dadas para la utilización de los datos y, en particular, al investigador principal del proyecto. El cuestionario de investigación del que parte el análisis se halla estructurado 
con una disposición interna multitemática que integra, entre otras cuestiones, variables destinadas a registrar la implicación, la valoración y las acciones desplegadas por los sujetos participantes en su experiencia dentro de las reuniones del presupuesto participativo. En relación con el diseño muestral, la investigación siguió una lógica exploratoria, dada la ausencia de marcos muestrales y de datos precedentes sobre la estructura sociodemográfica de los participantes en los distintos municipios. De esta manera, se determinaron cuotas abiertas en relación con las variables género y edad para un muestreo no probabilístico, componiendo una muestra final con un tamaño de 1.881 casos, de los que, a España (que constituye el marco de estudio en este texto), corresponde una muestra de 401 casos, distribuida en cuatro municipios (Sevilla, Ferrol, Novelda y Torreperogil). La selección de estos municipios responde a la necesidad de la encuesta de operar con fórmulas organizativas de experiencias de procesos enmarcados en los llamados «modelos participativos» de presupuestos participativos (Ganuza y Francés, 2012), que son los únicos que se plantean bajo la premisa de celebración de reuniones ciudadanas deliberativas abiertas a la participación de forma individual, lo que hace posible el registro de cuestionarios de forma presencial. La implementación del trabajo de campo fue homogénea en todos los municipios donde se llevó a cabo la encuesta: cuestionarios anóminos, validados por pretest y autoaplicados con la asistencia técnica del personal responsable de los procesos al finalizar las asambleas del presupuesto participativo.

\section{Análisis y discusión de resultados. La valoración de las condiciones de la deliberación}

El primer aspecto que podríamos considerar en el análisis de los resultados parte de la idea de que la concurrencia de los sujetos en un espacio público habilitado para la toma de decisiones comunes debería favorecer en los participantes el desarrollo de nociones como la responsabilidad social, la solidaridad o la confianza con respecto a otros individuos o grupos, además de orientar el debate hacia consensos que aportaran un interés general en la legitimidad de los resultados (Delli et al., 2004: 318). Incluso, aunque no se alcance finalmente un acuerdo acerca de los intereses comunes, como afirma Gould (1989), a través de estas interacciones, se promueve cuando menos cierto sentido de la reciprocidad. Dicho esto, podemos intuir que, en un proceso como el del presupuesto participativo, el concurso simultáneo de intereses distintos y divergentes genera un pluralismo que dista mucho de ser residual. Con frecuencia, las preferencias y los derechos esgrimidos por iniciativas de unos ciudadanos entran en conflicto con los derechos o los intereses de otros grupos de ciudadanos. Así, por ejemplo, una propuesta que plantee el cerrar un vial al tráfico rodado en una zona determinada de la ciudad para favorecer el esparcimiento público de los peatones puede colisionar con los intereses de los comerciantes, que ven en esta iniciativa una disminución potencial del volumen de ventas, al reducirse la afluencia. Otro ejemplo podría ilustrarse ante la situación que se plantea cuando existe un solar de propiedad pública y se abre un debate acerca 
de sus posibles usos. Allí donde unos ven en ese espacio un lugar ideal para construir un equipamiento deportivo, otros pueden ver en él la oportunidad para ampliar las zonas verdes de la ciudad o para situar nuevas dependencias públicas. Podríamos encontrar muchísimos ejemplos, pero basten estos para comprender que, cuanto más se abre el proceso participativo a los actores sociales, mayores probabilidades existen de que se presenten profundas divergencias en la interacción deliberativa. La razón esencial de ello, como comenta Máiz (1996), se debe a que las preferencias individuales no se constituyen jamás sobre intereses generalizables, como si existiera un sustrato común que hiciera ver a los sujetos cuál es el interés predominante y garantizara el acuerdo unánime.

La comunicación y la deliberación producen consenso, pero también, ineludiblemente, conflicto (Mansbridge, 1993; Prono, 2010: 113), y tanto la disensión como el conflicto contribuyen en gran medida al proceso deliberativo tomándolos como campo de acción (Maliandi, 2006: 218), puesto que obliga a los participantes a desplazarse desde sus preferencias singulares hacia la comprensión de una concurrencia de intereses propios y ajenos, dando lugar a una pluralidad ampliada, en referencia a la pluralidad de perspectivas, recíprocas, que configuran el entorno del proceso de deliberación. La pluralidad ampliada contribuye, de este modo, a conseguir que, puesto que rara vez se dispone de consenso al principio de los procesos, sea necesario realizar un esfuerzo por el reconocimiento mutuo, por desarrollar un ejercicio de empatía y solidaridad hacia los intereses de los demás, a la vez que a buscar modos democráticos en los comportamientos para desplegar la interacción deliberativa en las mejores condiciones posibles. Si, para contextualizar esta cuestión, utilizamos los datos obtenidos por el IESA en su estudio en el que se encuestaba a los participantes de varias experiencias de presupuestos participativos en España, los resultados quedan consignados en la tabla 1.

Los datos apuntan hacia una percepción con relativos sesgos que permiten adivinar perfiles. Tanto la edad como la ideología presentan diferencias internas significativas que indican que los participantes más jóvenes y de izquierdas hacen una valoración más positiva de las condiciones del debate en el seno de las reuniones ciudadanas dentro del presupuesto participativo, tanto en términos de democracia interna como de solidaridad en la interacción entre participantes. Estas diferencias, en cambio, no son significativas en lo relativo al sexo ni al nivel educativo. Pero, en términos generales, a partir de los resultados de las valoraciones medias de los participantes, podemos hablar de una evaluación favorable dentro las condiciones clásicas para definir un entorno deliberativo adecuado. Dichas condiciones (Mansbridge et al., 2010: 65) abarcan las premisas de que el debate esté abierto a todas aquellas personas como afectadas por las decisiones a tomar en un ambiente no coercitivo de que los participantes perciban que tienen oportunidades parecidas para influir en el proceso (para el caso del presupuesto participativo que las reuniones sean juzgadas como democráticas) y de que el entorno permita una traslación de preferencias e intereses entre las personas que interactúan en el debate (para 
Tabla 1. Calificaciones medias de los deliberantes en las asambleas del presupuesto participativo en las que han participado en relación con el grado de solidaridad y democracia interna percibido

\begin{tabular}{|c|c|c|}
\hline Perfiles & $\begin{array}{l}\text { Solidaridad percibida } \\
\text { en la reunión } \\
(0=\text { manipulada } / \\
7=\text { democrática })\end{array}$ & $\begin{array}{l}\text { Democracia interna percibida } \\
\text { en la reunión } \\
\text { ( } 0=\text { insolidaria } / \\
7=\text { solidaria) }\end{array}$ \\
\hline \multicolumn{3}{|l|}{ SEXO } \\
\hline $\begin{array}{l}\text { Hombre } \\
\text { Mujer }\end{array}$ & $\begin{array}{l}4,66 \\
4,64\end{array}$ & $\begin{array}{l}4,35 \\
4,39\end{array}$ \\
\hline $\mathrm{F}$ de ANOVA & 0,008 & 0,031 \\
\hline Sig. & 0,931 & 0,860 \\
\hline \multicolumn{3}{|l|}{ EDAD } \\
\hline $\begin{array}{l}\text { Menos de } 45 \text { años } \\
45 \text { o más años }\end{array}$ & $\begin{array}{l}4,94 \\
4,65\end{array}$ & $\begin{array}{l}4,65 \\
4,21\end{array}$ \\
\hline F de ANOVA & 3,781 & 4,687 \\
\hline Sig. & 0,048 & 0,031 \\
\hline \multicolumn{3}{|l|}{ NIVEL EDUCATIVO } \\
\hline $\begin{array}{l}\text { Sin estudios } \\
\text { Estudios primarios inacabados } \\
\text { Estudios primarios } \\
\text { Estudios secundarios } \\
\text { Estudios universitarios }\end{array}$ & $\begin{array}{l}4,56 \\
4,27 \\
4,49 \\
4,87 \\
5,01\end{array}$ & $\begin{array}{l}4,64 \\
4,14 \\
4,17 \\
4,61 \\
4,48\end{array}$ \\
\hline F de ANOVA & 1,852 & 1,086 \\
\hline Sig. & 0,118 & 0,363 \\
\hline \multicolumn{3}{|l|}{ IDEOLOGÍA } \\
\hline $\begin{array}{l}\text { Izquierda } \\
\text { Centro } \\
\text { Derecha }\end{array}$ & $\begin{array}{l}5,04 \\
4,58 \\
3,95\end{array}$ & $\begin{array}{l}4,60 \\
4,55 \\
3,53\end{array}$ \\
\hline F de ANOVA & 6,653 & 5,970 \\
\hline Sig. & 0,001 & 0,003 \\
\hline
\end{tabular}

En negrita, diferencias significativas para el valor $p<0,05$.

Fuente: IESA Estudio E1106.

$N=349$.

el caso del presupuesto participativo que las reuniones sean juzgadas como solidarias). Los datos de las experiencias analizadas confirman estas ideas: el $75,4 \%$ de los participantes en la encuesta se aproximan a la etiqueta de que las reuniones acontecidas fueron democráticas y casi el $71,5 \%$ se sitúa cercano a la idea de que las reuniones fueron solidarias.

En cualquier caso, el juego de traslación de intereses que proporciona la percepción de democracia interna y solidaridad entre los sujetos participantes no es unívoca ni necesariamente natural dentro del proceso deliberativo. Para ello, es necesario recurrir a la negociación de unas reglas mínimas del juego participativo, reglas que permitan la transformación cualitativa de las preferencias iniciales para alcanzar consensos y que, en el fondo, constituyen el rasgo carac- 
Gráfico 1. Evaluación subjetiva de los deliberantes en el presupuesto participativo y dimensiones del debate colectivo

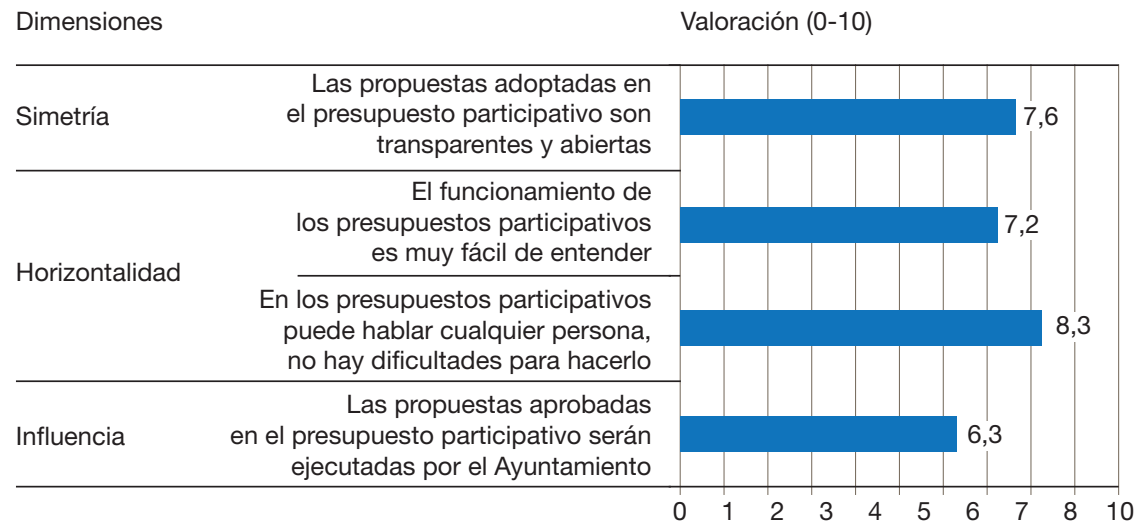

Fuente: IESA Estudio E1106.

$N=401$.

Nota: las pruebas realizadas a través del estadístico F de ANOVA con las variables sexo, edad y nivel educativo muestran en todos los casos una significación $>0,05$, lo que confirma las hipótesis de independencia de los ítems analizados en relación con dichas variables.

terístico de este tipo de interacción política (Prono, 2010: 114). Reglas que, en el caso de los presupuestos participativos, no constituyen un marco normativo apriorístico, sino que se construyen ad hoc a partir de una premisa fundamental en la mayoría de experiencias: la creación de un marco normativo que asegure relaciones simétricas y horizontales entre los participantes, que concurren en el espacio participativo con la única limitación de la disponibilidad de recursos existentes para las decisiones y la viabilidad práctica de los acuerdos que se alcancen. Cabe preguntarse a este respecto si los participantes en un proceso ambicioso en términos deliberativos como es el presupuesto participativo valoran, tras su experiencia asamblearia, de forma positiva estas premisas de simetría y horizontalidad en la práctica de su interacción deliberativa tras el debate. Los resultados obtenidos a partir de la elaboración de dimensiones (gráfico 1), de acuerdo con los datos del estudio realizado por el IESA, nos muestran que, efectivamente, las condiciones del debate son juzgadas acordes a estas dos ideas por parte de los participantes, independientemente de su sexo, edad o nivel educativo, lo que confiere adicionalmente a este mecanismo un valor significativo carente de importantes sesgos poblacionales en el abordaje pragmático de estas cuestiones dentro de la práctica deliberativa.

Los resultados determinan un amplio apoyo a la idea de la simetría en la información que circula dentro del proceso («Las propuestas adoptadas en el presupuesto participativo son transparentes y abiertas») y existe una valoración positiva de la horizontalidad en su funcionamiento ( $\mathrm{El}$ funcionamiento de los presupuestos participativos es muy fácil de entender» $\mathrm{y}$ «En los presupuestos 
Tabla 2. Correlaciones bivariadas entre las variables vinculadas a condiciones en que se ha desarrollado el debate del presupuesto participativo y las variables relacionadas con la evaluación subjetiva del proceso

\begin{tabular}{|c|c|c|c|}
\hline & & $\begin{array}{l}\text { Punto de vista de la } \\
\text { reunión: manipulada / } \\
\text { democrática }\end{array}$ & $\begin{array}{l}\text { Punto de vista de la } \\
\text { reunión: insolidaria / } \\
\text { solidaria }\end{array}$ \\
\hline $\begin{array}{l}\text { Las propuestas adoptadas en } \\
\text { el presupuesto participativo son } \\
\text { transparentes y abiertas }\end{array}$ & $\begin{array}{l}\text { C Pearson } \\
\text { Sig. } \\
N\end{array}$ & $\begin{array}{l}0,225^{\star *} \\
0,000 \\
337\end{array}$ & $\begin{array}{l}0,212^{\star *} \\
0,000 \\
316\end{array}$ \\
\hline $\begin{array}{l}\text { El funcionamiento de los } \\
\text { presupuestos participativos } \\
\text { es muy fácil de entender }\end{array}$ & $\begin{array}{l}\text { C Pearson } \\
\text { Sig. } \\
N\end{array}$ & $\begin{array}{c}0,248^{\star *} \\
0,000 \\
319\end{array}$ & $\begin{array}{l}0,212^{\star *} \\
0,000 \\
340\end{array}$ \\
\hline $\begin{array}{l}\text { En los presupuestos participativos } \\
\text { puede hablar cualquier persona, } \\
\text { no hay dificultades para hacerlo }\end{array}$ & $\begin{array}{l}\text { C Pearson } \\
\text { Sig. } \\
N\end{array}$ & $\begin{array}{l}0,196^{* *} \\
0,000 \\
340\end{array}$ & $\begin{array}{c}0,130^{*} \\
0,020 \\
318\end{array}$ \\
\hline $\begin{array}{l}\text { Las propuestas aprobadas en } \\
\text { el presupuesto participativo serán } \\
\text { ejecutadas por el Ayuntamiento }\end{array}$ & $\begin{array}{l}\text { C Pearson } \\
\text { Sig. } \\
N\end{array}$ & $\begin{array}{c}-0,222^{* *} \\
0,000 \\
338\end{array}$ & $\begin{array}{c}-0,184^{* *} \\
0,001 \\
317\end{array}$ \\
\hline
\end{tabular}

** La correlación es significativa al nivel 0,01 (bilateral).

* La correlación es significante al nivel 0,05 (bilateral).

Fuente: IESA Estudio E1106. $++^{\circ}+{ }^{\circ \circ}$

participativos puede hablar cualquier persona, no hay dificultades para hacerlo»). En gran medida, podría pensarse que este apoyo evaluativo al proceso debe ser consistente con la apreciación que los sujetos hacen de las condiciones en que haya acontecido el debate colectivo. En otras palabras, cabría esperar cierta concordancia entre la valoración final del proceso participativo y la percepción de las condiciones deliberativas en que se han desarrollado las reuniones ciudadanas. La tabla 2 muestra los resultados empíricos en torno a esta cuestión.

Aunque con unos coeficientes no excesivamente elevados en los estadísticos de asociación, los resultados muestran correlaciones significativas entre las variables vinculadas a las condiciones en que se ha desarrollado el debate del presupuesto participativo y aquellas que fijan la evaluación subjetiva del proceso por parte de los participantes. Ello nos indica que tanto la percepción del entorno deliberativo como la evaluación de los logros procedimentales y pragmáticos del proceso se hallan ligados, y nos habla de la constatación de la existencia de un marco adecuado para la interacción deliberativa.

$\mathrm{Si}$, a través de la negociación y la deliberación, queda institucionalizado (que no anulado) el conflicto, con todo lo problemático que esto resulta, implícitamente estamos reconociendo en ocasiones la imposibilidad de alcanzar consensos en torno a determinados temas o intereses. Con frecuencia, en las reuniones ciudadanas del presupuesto participativo, se impone la necesidad de aceptar decisiones no consensuadas o, lo que es lo mismo, decisiones mediante criterio de mayorías versus criterio de unanimidad. Aquí cobran una 
Tabla 3. Valoración de las condiciones deliberativas en las experiencias españolas de presupuestos participativos por parte de los participantes

¿Piensa Ud. que tenía suficiente información como para participar en el debate de los presupuestos participativos?

¿Piensa Ud. que ha habido suficiente tiempo para debatir?

\begin{tabular}{lrr}
\hline Sí & $71,1 \%$ & $72,5 \%$ \\
No & $28,4 \%$ & $26,4 \%$ \\
NC & $0,5 \%$ & $1,1 \%$ \\
\hline
\end{tabular}

Fuente: IESA Estudio E1106.

$N=401$.

Nota: las pruebas de asociación bivariables realizadas a través de chi cuadrado con las variables sexo, edad, nivel educativo e ideología aportan en todos los casos una significación $>0,05$, lo que confirma las hipótesis de independencia de los ítems analizados en relación con dichas variables.

especial atención los tiempos o ritmos destinados al proceso deliberativo. Al abrir dispositivos no consensuados para la toma de decisiones colectivas, es posible incurrir en el peligro de que el acto de la decisión anule o subordine el proceso de reflexión deliberativa. Esta situación provocaría que, en lugar de la búsqueda de consenso a través del debate colectivo, se recurriera rápidamente al mecanismo de decisión como forma de resolver el conflicto de intereses, perdiendo así la deliberación su potencial reflexivo. La inercia vendría representada por el principio de «si no hay acuerdo, a votar», recurso que observamos si no con frecuencia al menos sí eventualmente en los procesos participativos. Es cierto que, en ocasiones, es imposible llegar a consensos totales en torno a las decisiones, pero si la decisión por mayorías se eleva como mecanismo rápido y automático para dirimir divergencias, el proceso participativo queda abocado a un escenario en el que la experiencia deliberativa, reflexiva y participativa reproduciría gran parte de las limitaciones presentes en la lógica de la democracia representativa, estableciendo como único mecanismo para dirimir cuestiones el juego de mayorías y empobreciendo el potencial que incorpora la interacción comunicativa. Por ello, los tiempos dedicados a la reflexión conjunta previa a la decisión son, en ocasiones, tanto o más importantes que las decisiones finalmente adoptadas. En este sentido, la encuesta realizada por el IESA revela que las experiencias españolas de presupuestos participativos son juzgadas por los participantes como un marco que permite el despliegue de información y de tiempos suficientes para la deliberación colectiva, independientemente de variables sociodemográficas como el sexo, la edad, el nivel educativo o la ideología (tabla 3).

De acuerdo a ello, el paradigma ya no sería el mercado (de ideas) y la búsqueda de apoyos, sino el diálogo, y la concepción dialógica imagina la política como respuesta ante cuestiones de valor y no simplemente como una cuestión de preferencia a resolver. Interacción simétrica, comunicación, información, tiempo e identificación y reconocimiento de la diferencia suponen, por lo tanto, los anclajes de partida y llegada en una visión deliberativa y participativa de la democracia en la figura del presupuesto participativo y otras de naturaleza 
similar, algo que, en este caso, alcanza cotas apreciables de valoración en los participantes.

La garantía de condiciones para el debate deliberativo trae a la discusión del análisis a otro actor fundamental dentro de los procesos participativos: las instituciones y el rol que desempeñan en relación con los participantes. Aquí las investigaciones realizadas sugieren que la invitación a participar por parte de las autoridades desarrolla un interés que, de otra forma, posiblemente no habría aparecido (Stewart, 2001: 84). Si se asume la acción participativa y deliberativa en la lógica de funcionamiento institucional, se permitirá la incorporación de unos resultados que difícilmente son alcanzables a partir de la mera agregación de intereses ciudadanos o mediante acciones participativas al margen de las instituciones. Pero, además, para el componente institucional, estos procesos inciden como mínimo en la legitimación de la acción pública (Font y Blanco, 2001: 225; Halvorsen, 2003: 541; Newman et al., 2004: 210; Fung, 2006; Abels, 2007: 111), y más allá en otro nivel sujeto todavía a discusión, la cuestión de la mayor efectividad y eficiencia de las políticas cuando estas parten de acuerdos resultantes de procesos abiertos a la deliberación participativa (Simrell et al., 1998: 319; Subirats, 2001: 40; Lowndes y Sullivan, 2004; Burton, 2009: 266). Parece, por lo tanto, clara la incidencia de la estructura institucional en los procesos participativos, y por ello queda justificado el hecho de que cualquier estudio de la participación ciudadana en su vertiente institucional pase por analizar la relación dialéctica entre la acción participativa como resultado de la interacción deliberativa y la estructura de oportunidades que brinda el sistema al entramado político-institucional para ello (Richardson, 1995; Benedicto, 2004: 229).

La potencialidad de la lógica participativa no está reñida con una institucionalización que otorgue carta de garantías a la plasmación práctica de los resultados que se van produciendo a partir de la deliberación colectiva. Esta institucionalización se torna incluso necesaria si, desde los gobernantes, existe una verdadera voluntad en el asentamiento temporal de una figura como el presupuesto participativo, puesto que, en las instituciones, reside la capacidad última (ya sea legal o ejecutiva en muchos casos) para implementar los acuerdos alcanzados por los participantes. Si no existe confianza por parte de los sujetos implicados en la respuesta y el apoyo del entramado institucional, o si el impacto pragmático del proceso de participación es muy limitado en relación con la acción pública, difícilmente desarrollarán una implicación activa o, como ocurre en muchos casos, acabará produciéndose una sensación de frustración por las expectativas incumplidas. En este sentido, y para la experiencia del presupuesto participativo, como hemos visto por los resultados del gráfico número 1, pese a que los participantes creen ampliamente que las decisiones adoptadas en el debate colectivo serán ejecutadas por la corporación municipal, este constituye el indicador evaluativo donde las personas se muestran más escépticas. Más allá, los datos obtenidos revelan también el reducido carácter transformador de este dispositivo como herramienta de planificación local a juicio de los participantes (tabla 4), algo por otra parte ligado, como se aprecia en distintos estudios, a la percepción del compromiso 
Tabla 4. Respuestas de los deliberantes en el presupuesto participativo a la pregunta: ¿Qué dos problemas soluciona efectivamente el presupuesto participativo a día de hoy?

\begin{tabular}{lcc}
\hline & Sí & No \\
\hline Resolver proyectos urbanos a escala del barrio & $64,4 \%$ & $35,6 \%$ \\
Resolver problemas que afectan a toda la ciudad & $23,2 \%$ & $76,8 \%$ \\
Problemas estratégicos que afectan al futuro de la ciudad & $11,6 \%$ & $88,4 \%$ \\
Problemas de desigualdad social & $14,6 \%$ & $85,4 \%$ \\
Mejorar el funcionamiento de la Administración & $10,7 \%$ & $89,3 \%$ \\
\hline
\end{tabular}

Fuente: IESA Estudio E1106.

$N=233$.

institucional por parte de los sujetos y al grado de recursos sometidos a debate en los procesos (Allegretti et al., 2012; Allegretti et al., 2011).

El compromiso institucional es una de las demandas que frecuentemente se repiten en las dinámicas deliberativas internas dentro de los presupuestos participativos. Los ciudadanos deben aportar al proceso una cantidad importante de esfuerzos y dedicación personal, y, como contraparte, exigen de las instituciones ciertas garantías de que esos esfuerzos y tiempos obtendrán el debido respaldo, a la vez que ambición para convertir al proceso participativo en un verdadero mecanismo transformador y de planificación en la política pública local. Desde el lado de la ciudadanía, la democracia como deliberación debe aunar la doble vertiente de agregación e integración. Desde el nivel gubernamental, la institucionalización de los procedimientos supone una clave fundamental para sostener la credibilidad de este enfoque de la democracia. Experiencias como el presupuesto participativo carecen de sentido sin una dimensión institucional. En el fondo, la pregunta a realizar es si es posible un proceso deliberativo institucionalizado en el cual los participantes pueden influir directamente sobre la acción pública. Y, en este sentido, varios autores (entre otros Baiocchi, 2005: 144; Ganuza y Francés, 2012: 299) han identificado como factor clave las condiciones de las instituciones locales para explicar la calidad que despliegan las experiencias y evitar sesgos deliberativos, tanto en la entrada del proceso (captando un pluralismo sociodemográfico suficientemente representativo) como en su desarrollo (respetando tiempos y garantizando una distribución equitativa de oportunidades deliberativas) y en su salida (ejecutando las propuestas debatidas por la ciudadanía), y más allá, aportando garantías de que los posibles cambios de color político en los gobiernos no afectarán a la validez de los acuerdos alcanzados por los participantes (López y Pineda, 2013: 280).

\section{Conclusiones}

El análisis desarrollado a lo largo del texto y su contrastación empírica nos ha perseguido identificar cuestiones clave para la articulación de un espacio deliberativo y participativo orientado al debate sobre decisiones públicas. Tres son los elementos que han sido considerados en este ámbito: las condiciones en 
que se despliega la deliberación, la relación entre estas y la valoración cognitiva y procesual que realizan los participantes de su experiencia, así como los nexos entre ambas cuestiones y la percepción que tienen los ciudadanos en relación con el rol del entramado institucional en todo el proceso.

Por parte de la ciudadanía, hemos podido comprobar que la valoración de las condiciones deliberativas en las que se desarrollan las reuniones ciudadanas dentro del seno de los presupuestos participativos son juzgadas como satisfactorias, tanto en términos de apreciación de la solidaridad existente como en relación con la democracia interna percibida en estas, si bien variables como la edad y la ideología desempeñan un papel diferencial en este aspecto. Estas condiciones se asocian positivamente con la evaluación final que hacen los participantes del presupuesto participativo en términos de horizontalidad y simetría como principios articuladores de la deliberación colectiva. En este sentido, podemos concluir diciendo que las experiencias analizadas del presupuesto participativo ofrece un marco adecuado para la interacción deliberativa en presencia de unos participantes plurales. Los clásicos sesgos participativos procedentes de los perfiles sociodemográficos como el sexo, la edad o el nivel educativo no ofrecen, en este ámbito de análisis, diferencias significativas en la valoración de la calidad deliberativa de las reuniones ciudadanas, lo cual facilita los aspectos dinámicos de la interacción social.

La información y el tiempo disponible para alcanzar acuerdos constituyen dos claves más a añadir en la configuración de las condiciones deliberativas en procesos de este tipo orientados a las decisiones públicas. El objetivo, en grandes palabras, lo constituiría el aumento de la capacitación y la competencia de la ciudadanía, así como la mejora de la calidad de la información para la toma de decisiones. A la luz de los resultados del análisis, el encauzamiento de la decisión no subordina, a juicio de los participantes, el proceso de reflexión y deliberación, que contempla tanto el intercambio de información en el sentido vertical y horizontal como la depuración deliberativa óptima de esta por parte de los sujetos a través del tiempo y de la información disponibles para alcanzar una formación cualificada de los intereses propios y ajenos. Ello nos deriva al debate incómodo del rol de las instituciones en el despliegue de este tipo de procesos y su vinculación a las decisiones finalmente alcanzadas en él. Aquí se muestran imprescindibles no solo las garantías ejecutivas (la aceptación e implementación de la decisión final), sino también las metodológicas, para lo cual, en el caso del presupuesto participativo, algunas experiencias se han pertrechado de toda una normativa que garantice una deliberación lo más capacitada posible (Avritzer, 2005: 62), a través, fundamentalmente, del respeto de tiempos y plazos, asistencia técnica para clarificar los condicionantes de las decisiones, dotación de los recursos económicos e informativos necesarios o requerimientos para la adecuada representación sociodemográfica de toda la población a lo largo del proceso. Los resultados empíricos presentados en el análisis de la figura del presupuesto participativo, a partir de las garantías mencionadas, apuntan, en este sentido, a la posibilidad pragmática de deliberación entre un espectro heterogéneo de intereses, alimentando así la concepción tradicional de la participación mediante la incorporación de elementos propios 
de la teoría deliberativa. La conclusión se encamina así hacia la factibilidad de combinación de sólidos componentes deliberativos insertados en procesos prácticos ambiciosos en términos de participación. Ello, en condiciones ideales, posibilita la superación de las limitaciones propias de los minipúblicos tradicionalmente objeto de las experiencias deliberativas, e incorpora cotas apreciables de horizontalidad entre la ciudadanía y las instituciones en la toma de decisiones públicas, cuestión, por otra parte, ya constatada en otros estudios sobre presupuestos participativos (Sintomer et al., 2011: 15), pero que continúa siendo un desafío en la dimensión pragmática de la democracia deliberativa.

\section{Referencias bibliográficas}

Abels, G. (2007). "Citizen involvement in public policy-making: Does it improve democratic legitimacy and accountability? The Case of pTA». Interdisciplinary Information Sciences [en línea], 13 (1), 103-116. $<$ http://dx.doi.org/10.4036/iis.2007.103>.

Allegretti, G. et al. (2012). Estudio comparativo de los presupuestos participativos en República Dominicana, España y Uruguay. Málaga: Diputación de Málaga.

Allegretti, G.; García, P. y PaÑo, P. (2011). Viajando por los presupuestos participativos: Buenas prácticas, obstáculos y aprendizajes. Málaga: Diputación de Málaga.

Avritzer, L. (2000). «Public deliberation at the local level: Participatory budgeting in Brazil». Paper delivered at the Experiments for Deliberative Democracy Conference. Wisconsin.

- (2005). «New public spheres in Brazil: Local democracy and deliberative politics». Revista Direito GV [en línea], número especial, 1, 55-74. <http://dx.doi.org/10.1111/j.1468-2427.2006.00692.x>.

Bächtiger, A.; Steenbergen, M. y Niemeyer, S. (2007). «Deliberative Democracy: An Introduction». Swiss Political Science Review [en línea], 13 (4), 485-496. $<$ http://dx.doi.org/10.1002/j.1662-6370.2007.tb00086.x>.

Baiocchi, G. (2005). Militants and Citizens. California: Standford University Press.

Baiocchi, G. y GanuZa, E. (2014). "Participatory Budgeting as if emancipation mattered». Politics \& Society [en línea], 42 (1), 29-50. $<$ http://dx.doi.org/10.1177/0032329213512978>.

Barber, B. (1988). The Conquest of Politics: Liberal Philosophy in Democratic Times. Princeton: Princeton University Press.

- (1998). «Democracia fuerte: Un marco conceptual: Política de la participación». En: Águila, R. del, Vallespín, F. et al. La democracia en sus textos. Madrid: Alianza, 281-296.

Barnes, M.; Newman, J. y Sullivan, H. (2006). «Discursive Arenas: Deliberation and the Constitution of Identity in Public Participation at Local Level». Social Movement Studies [en línea], 5 (3), 193-207. <http://dx.doi.org/10.1080/14742830600991487>.

Barreiro, F. (2012). «Ciudadanos y vecinos: Proximidad y participación ciudadana: Algunos dilemas no resueltos». Journal of Public Policies and Territories, 2, 1-9.

Bassoli, M. (2012). «Participatory Budgeting in Italy: An Analysis of (Almost Democratic) Participatory Governance Arrangements». International Journal of Urban and Regional Research, 36 (6), 1183-1203. $<$ http://dx.doi.org/10.1111/j.1468-2427.2011.01023.x>. 
Benedicto, J. (2004). «El cambiante escenario de la participación política: ¿Hacia una política participativa?». Zona Abierta, 106-107, 225-260.

Berger P. y Luckmann, T. (1993). La construcción social de la realidad. Buenos Aires: Amorrortu.

Beste, S. (2013). "Contemporary Trends of Deliberative Research: Synthesizing a New Study Agenda». Journal of Public Deliberation, 9 (2), 1-44.

Bohman, J. (1996). Public Deliberation: Pluralism, Complexity, and Democracy. Cambridge (MA): MIT Press.

- (1998). "The Coming of Age of Deliberative Democracy». The Journal of Political Philosophy [en línea], 6 (4), 400-425. <http://dx.doi.org/10.1111/1467-9760.00061>.

Burton, P. (2009). "Conceptual, Theoretical and Practical Issues in Measuring the Benefits of Public Participation». Evaluation [en línea], 15 (3), 263-284. <http://dx.doi.org/10.1177/1356389009105881>.

Cardoso, R.; Moreira, R. y Almeida, F. (2011). «Participation and Deliberation on the Internet: A Case Study on Digital Participatory Budgeting in Belo Horizonte». The Journal of Community Informatics, 7 (1-2).

Chambers, S. (2003). «Deliberative Democratic Theory». Annual Review of Political Science [en línea], 6, 307-326. <http://dx.doi.org/10.1146/annurev.polisci.6.121901.085538>.

Comen, J. y Fung, A. (2004). «Radical Democracy». Swiss Journal of Political Science, $10(4), 23-34$.

Colombo, A. (1998). Desobediencia civil y democracia directa. Madrid: Trama Editorial.

DeCaro, D. y Stokes, M. (2013). «Public Participation and Institutional Fit: A Social-Psychological Perspective». Ecology and Society [en línea], 18 (4), 40. <http://dx.doi.org/10.5751/es-05837-180440>.

Delli Carpini, M.; Cook, F. y Jacobs, L. (2004). «Public Deliberation, Discursive Participation and Citizen Engagement: A Review of the Empirical Literature». Review of the Empirical Literature: Annual Review of Political Science [en línea], 7 (1), 315-344. <http://dx.doi.org/10.1146/annurev.polisci.7.121003.091630>.

Dover, G. y Lawrence, T. (2010). «A Gap Year for Institutional Theory: Integrating the Study of Institutional Work and Participatory Action Research». Journal of Management Inquiry [en línea], 19 (4), 305-316. <http://dx.doi.org/10.1177/1056492610371496>.

Dryzex, J. (2000). Deliberative Democracy and Beyond: Liberals, Critics, Contestations. Oxford: Oxford University Press.

Dzur, A. (2008). Democratic Professionalism: Citizen Participation and the Reconstruction of Professional Ethics, Identity, and Practice. Pennsylvania: Pennsylvania State University Press.

ENGELKEN-Jorge, M. (2012). «Un balance de los giros empírico, sistémico y retórico de la teoría deliberativa». RIPS, 11 (4), 13-30.

Flynn, T. (2011). Debating Deliberative Democracy: How Deliberation Changes the Way People Reason. York: University of York. Department of Politics.

Font, J. y Blanco, I. (2001). «Conclusiones». En: Font, J. (coord.). Ciudadanos y decisiones públicas. Barcelona: Ariel.

Fung, A. (2004). Empowered Participation: Reinventing Urban Democracy. Princeton and Oxford: Princeton University Press. 
- (2006). «Varieties of Participation in Complex Governance». Public Administration Review [en línea], número especial, 66-75. <http://dx.doi.org/10.1111/j.1540-6210.2006.00667.x>.

Fung, A. y Wright, E. O. (2001). «Deepening Democracy: Institutional Innovations in Empowered Participatory Governance». Politics and Society [en línea], 29 (1), 5-41. <http://dx.doi.org/10.1177/0032329201029001002>.

- (2003). Deepening Democracy: Institutional Innovations in Empowered Participatory Governance. Londres: Verso.

Ganuza, E. y Baiocchi, G. (2012). «The Power of Ambiguity: How Participatory Budgeting Travels the Globe». Journal of Public Deliberation [en línea], 8 (2), artículo 8.

Ganuza, E. y Francés, F. (2012). «The Deliberative Turn in Participation: The Problem of Inclusion and Deliberative Opportunities in Participatory Budgeting». European Political Science Review [en línea], 4 (2), 283-302. <http://dx.doi.org/10.1017/s1755773911000270>.

- (2012). El círculo virtuoso de la democracia: Los presupuestos participativos a debate. Madrid: CIS.

Gould, C. (1989). Rethinking Democracy: Freedom and Social Co-operation in Politics, Economy, and Society. Cambridge: Cambridge University Press.

Gutmann, A. y Thompson, D. (2004). Why Deliberative Democracy? Princeton: Princeton University Press.

Habermas, J. (1991). "Cuestiones y contracuestiones». En: Guiddens, A. et al. Habermas y la modernidad. Madrid: Cátedra.

- (1998). «Derechos humanos y soberanía popular: Las versiones liberal y republicana». En: Águila, R. del y Vallespín, F. et al. La democracia en sus textos. Madrid: Alianza.

Halvorsen, K. (2003). «Assessing the Effects of Public Participation». Public Administration Review [en línea], 63 (5), 535-543. <http://dx.doi.org/10.1111/1540-6210.00317>.

Hansen, K. y Rostboll, C. (2012). "Deliberative Democracy». En: Isakhan, B. y Stockwell, S. (eds.). The Edinburgh Companion to the History of Democracy. Edimburgo: Edinburgh University Press.

Levine, P.; Fung, A. y Gastil, J. (2005). «Future Directions for Public Deliberation». Journal of Public Deliberation, 1 (1), artículo 3.

López, S. y Pineda, C. (2013). «Experiencias de presupuestos participativos en el País Valenciano: Análisis de variables que intervienen en su paralización». OBETS: Revista de Ciencias Sociales [en línea], 8 (2), 259-286. <http://dx.doi.org/10.14198/obets2013.8.2.03>.

Lowndes, V. y Sullivan, H. (2004). "Like a Horse and Carriage or a Fish on a Bicycle: How Well do Local Partnerships and Public Participation Go Together?». Local Government Studies [en línea], 30 (1), 51-73. <http://dx.doi.org/10.1080/0300393042000230920>.

Maliandi, R. (2006). Ética, dilemas y convergencias. Buenos Aires: Biblos.

Máiz, R. (1996). "Ni forum ni mercado: El déficit político de las dos teorías de la democracia». En: Porras, A. y Vega, P. de (ed.). El debate sobre la crisis de la representación política. Madrid: Tecnos.

Mansbridge, J. (1993). "Self-Interest and Political Transformation». En: Marcus, G. y Hanson, R. Reconsidering the Democratic Public. Pennsylvania: Pennsylvania State University Press. 
Mansbridge, J.; Bohman, J.; Chambers, S.; Estlund, D.; Føllesdal, A.; Fung, A.; Lafont, C.; Manin, B. y Martí, J. (2010). «The Place of Self-Interest and the Role of Power in Deliberative Democracy». The Journal of Political Philosophy [en línea], 18 (1), 64-100. <http://dx.doi.org/10.1111/j.1467-9760.2009.00344.x>.

Nanz, P. y Steffek, A. (2004). "Global Governance, Participation and the Public Sphere». Government and Opposition, 39 (2), 314-335. $<$ http://dx.doi.org/10.1111/j.1477-7053.2004.00125.x>.

Newman, J.; Barnes, M.; Sullivan, H. y Knops, A. (2004). «Public Participation and Collaborative Governance». Journal of Social Policy [en línea], 33(2), 203-223. <http://dx.doi.org/10.1017/S0047279403007499>.

O’Doherty, K. y Davidson, H. (2010). «Subject Positioning and Deliberative Democracy: Understanding Social Processes, Underlying Deliberation». Journal for the Theory of Social Behaviour [en línea], 40 (2), 224-245. <http://dx.doi.org/10.1111/j.1468-5914.2009.00429.x>.

O'Flynn, I. (2006). Deliberative Democracy and Divided Societies. Edimburgo: Edinburgh University Press.

Phillips, A. (1995). The Politics of Presence. Oxford: Clarendom Press.

Pineda, C. y Pires, V. (2008). «Formación y preparación de los actores del presupuesto participativo». Revista de Estudios Locales, 113, 54-75.

Prono, S. (2010). «La democracia deliberativa y el problema de su implementación práctica: Consideraciones críticas en torno al debate consenso vs. conflicto». Universitas: Revista de Filosofía, Derecho y Política, 12, 113-134.

Richardson, J. (1995). "The Market for Political Activism: Interest Groups as a Challenge to Political Parties». West European Politics [en línea], 18, 116-139. <http://dx.doi.org/10.1080/01402389508425060>.

Sanders, L. (1997). "Against Deliberation». Political Theory [en línea], 25 (3), 1-17. <http://dx.doi.org/10.1177/0090591797025003002>.

Simrell, C.; Feltey, K. y Susel, B. (1998). «The Question of Participation: Toward Authentic Public Participation in Public Administration». Public Administration Review [en línea], 58 (4), 317-326. <http://dx.doi.org/10.2307/977561>.

Sintomer, Y. et al. (2013). Participatory Budgeting in Asia and Europe: Key challenges of participation. Hampshire: Palgrave: McMillan.

Sintomer, Y.; Ganuza, E. y Röcke, A. (2011). Democracia participativa y modernización de los servicios públicos: Los presupuestos participativos en Europa. Amsterdam: Decouvere/TNI.

STEWART, J. (2001). «De la innovación democrática a la democracia deliberativa». En: Font, J. (coord.). Ciudadanos y decisiones públicas. Barcelona: Ariel.

Subirats, J. (2001). «Nuevos mecanismos participativos y democracia: Promesas y amenazas». En: FonT, J. (coord.). Ciudadanos y decisiones públicas. Barcelona: Ariel.

Talpin, J. (2011). Schools of Democracy: How Ordinary Citizens (Sometimes) Become Competent in Participatory Budgeting Institutions. Essex: ECPR.

Wampler, B. (2012). "Entering the State: Civil Society Activism and Participatory Governance in Brazil». Political Studies [en línea], 60 (2), 341-362. <http://dx.doi.org/10.1111/j.1467-9248.2011.00912.x>.

Young, I. (2000). Inclusion and Democracy. Oxford: Oxford University Press. 